\title{
PROTOTYPING OF COSMETIC PROSTHESIS OF UPPER LIMB USING ADDITIVE MANUFACTURING TECHNOLOGIES
}

\author{
Aleksandra Radosh'1, Wiesław Kuczko', Radosław Wichniarek', Filip Górski \\ Poznan University of Technology, Chair of Production Engineering and Management, Piotrowo 3 St., Poznan, \\ Poland, e-mail: ax.radosh@gmail.com,wieslaw.kuczko@put.poznan.pl, radoslaw.wichniarek@put.poznan.pl, \\ filip.gorski@put.poznan.pl
}

Received: 2017.05.15

Accepted: 2017.08.01

Published: 2017.09.03

\begin{abstract}
This paper describes prototyping of an individualized cosmetic arm prosthesis. Aim of the studies was to obtain an anatomically correct, lightweight prosthesis. It was accomplished using additive manufacturing technology of fused deposition modeling. The data was obtained by 3D scanning. An experimental concept of dual extrusion of two different materials was applied - the prosthesis was divided into an elastic shell and a rigid core, manufactured in one process. Obtained results were positive.
\end{abstract}

Keywords: upper limb, additive manufacturing, cosmetic prosthesis, medical prototype

\section{INTRODUCTION}

Lack of one or two of upper limbs is a significant limitation of human movement performance. This problem concerns a large group of people and can be a result of both birth defects and amputations. Birth limb defects (so-called birth amputations) are results of disturbances occurring in fetal life, such as stopping of growth or damage of fetus. As regards the amputations, the most frequent causes are [14]:

- vascular diseases and complications - approx. $65 \%$,

- diabetes and its complications - approx. $20 \%$,

- injuries - approx. 11,5\%.

Present level of technological advancement allows manufacturing prostheses of upper limbs, which can replace the lacking limb, to a certain degree. Their functions may be purely visual (cosmetic prostheses), but also fully operational - controlled mechanically or electronically (so-called bionic prostheses). For an average patient, a problem in accessing these devices is their price, which is proportional to technological advancement and quality of production of a given prosthesis.
It is also important, that there is a certain work and time required from the patient, to properly fit the prosthesis to his body shape. As certain studies indicate, there is often a problem in mutual communication between a patient and a prosthetist and it can negatively affect final satisfaction of use of a given prosthesis [11]. A prosthesis, as an artificial replacement of a missing body part, is manufactured in several steps - their total realization time can take, depending on the particular prosthesis type, between one week and 3 months. For the standard cosmetic prostheses, subsequent steps are as following [10]:

- Evaluation of amputation and patient physical possibilities.

- Production of cast of a stump (the so-called negative) out of calcined gypsum or making a digital scan.

- Making body measurements and making a model of a stump (the so-called positive).

- Heating a sheet of thermoplastic material and forming it in vacuum around the positive.

- Fitting the test socket and applying corrections for patient comfort.

- Manufacturing of final prosthesis, formed similarly as the test socket. 
As an attempt to solve or reduce scale of the above mentioned problems and limitations, it is proposed to use low-cost additive manufacturing technologies, also known as the 3D printing. They allow relatively quick obtaining of products of complex geometry, such as anatomic shapes. The greatest benefits of additive manufacturing, widely used, for example, in automotive and machine industry, are achieved with prototype and single piece production [4]. Production of prostheses adjusted to anthropometric features of particular patient's body is exactly of this character. What's more, additive manufacturing combined with the reverse engineering approach allows less problematic (in comparison to the traditional approach) processing, storing and transferring of anthropometric data, what makes it easier to repair prostheses - manufacturing of parts, which are worn out or damaged.

There are many different methods of additive manufacturing. The authors of this work selected one of the most widespread method - Fused Deposition Modelling (FDM), which also is the most economically justified one [3]. It allows manufacturing products out of materials, which can have contact with the human body, are not toxic and ensure good strength and dimensional and shape accuracy $[5,2]$.

Technical capabilities of 3D printers may prove insufficient in printing large-sized objects, in which case the element is divided into smaller elements printed separately and assembled to form the desired structure. In given circumstances one of the methods applied is adhesive joining [9]. The proper surface energy is a prerequisite to obtain strong adhesive bond [8], particularly of polymer adherents.

This paper presents a process of design and manufacturing of a prototype prosthesis for a female 23-year old patient, born without her left forearm, with remaining functional elbow joint. Assumption of the presented work was to obtain a prosthesis as comfortable as the one currently at disposal of the patient, but with higher strength and maintaining as low costs of the whole process as possible.

\section{THEORETICAL INFORMATION}

As a basis for development of project of a new prosthesis, an existing prosthesis owned by the patient was evaluated. Current cost of manufacturing of a very basic variant of such a cos- metic prosthesis in Poland is no less than 6000 PLN and depending on quality of used materials it can be many times higher. It means that a prosthesis is three times as expensive as the minimal salary, which illustrates problem with availability of such a solution for an average patient. Even if partial refund is possible via the National Health Fund, the patient still has to cover most of the costs. As an additional cost, a need for the patient to visit the prosthesis producer several times must be assumed, as these companies are usually located in bigger cities. Moreover, manufactured prosthesis wears off periodically and must be replaced after few years of use. In case of children patients, necessity of replacing the prosthesis is usually more frequent, which is related to growing up and changing body shape.

The authors have also analyzed projects of upper limb prostheses, which were realized earlier by other researchers. There are many projects of mechanically or electronically controlled prostheses, which are aimed at ensuring the highest accessibility for the patients, among other things by application of low-cost additive manufacturing technology of FDM. The most interesting projects are as following [7, 16, 15]:

- Robohand - open source project, directed to children without fingers. Product is controlled mechanically and almost $100 \%$ of the parts (except strings, Velcro straps and screws) are made using the FDM technology.

- Cyborg Beast - prosthesis built using the FDM device, out of ABS and PLA materials. Based on the RoboHand, enhanced with two-string tightening system - elastic and rigid elements. Current cost of materials necessary for manufacturing such a prosthesis is approx. 200 PLN and time of assembly of all the elements is 150 minutes.

- Limbitless Arm - bionic prosthesis, manufactured additively and equipped with electrical actuators and controllers. Control of the prosthesis is carried out by electrical muscle signals from patient's arm.

Additionally, it must be mentioned that FDM is also applied in manufacturing of other orthopedic equipment, used in human upper limbs. An example can be the Spiderhand device - a hand helping device for persons after strokes and spinal injuries. Operational principle is similar to the RoboHand, it transfers wrist movements into movement of fingers, but is attached to live fingers of a patient who is unable to move them on his own [13]. 


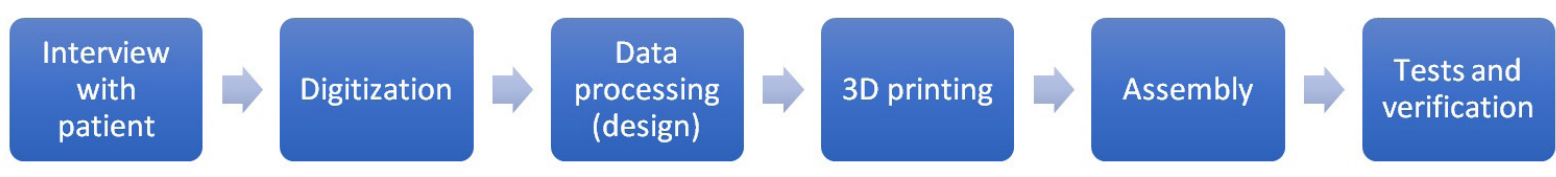

Fig. 1. Scheme of building the prosthesis

The build materials ABS and PLA (thermoplastic polymers) have good mechanical properties, but do not resemble human body, neither in appearance nor touch. That is why it is proposed to use extra elastic materials, such as the NinjaFlex [12].

Another common part of the above mentioned projects is lack of ideal fitting of the part, which is directly in contact with the patient body. The above mentioned products are parametrized, so their geometry can be changed to some degree by values of main dimensions. However, obtaining full representation of shapes compatible with anthropometric data of a patient requires using additional technologies. In case of the cosmetic prostheses not only the comfortable fitting, but also the visual aspect is important for the patient [1]. In case of patients having one of the limbs, a mirror image of this limb can be used for making the prosthesis. Gathering the required data is possible using various technologies of medical imaging. In literature, there are elaborations presenting scanning of the patient body using structural light [6]. In a similar way, plaster casts of patient body parts can be scanned. This is a process which is longer than direct scanning of a patient, but it allows obtaining data, that are not burdened with errors resulting from involuntary movement of the living patient during the scanning process.

\section{PRACTICAL STUDIES}

To build a prototype of customized hand and forearm prosthesis, a couple of tasks had to be performed, schematically presented in Fig. 1. First task was interview with the patient, to identify and record requirements. Then, anatomical features of the patient had to be digitized and the data had to be processed. Consequently, the prosthesis elements were manufactured according to the prepared design and their final assembly was made. Finally, the prosthesis was verified by a professional prosthetist.

Requirements defined by the patient were clear. The prosthesis should have esthetic looks, resembling a real hand, additionally it should be strong enough to enable supporting body on it, without fear of damaging it. The patient agreed to use a healthy limb as a pattern for designing the prosthesis. Because of size of the forearm, the authors were unable to make a casting, that is why this body part was scanned directly on the patient. Digital data from the forearm was of worse quality than data from the castings, which is why their further processing took much more time.

Gathering of anthropometric data was performed using the 3D ROMER Absolute Arm scanner. In case of the stump and hand of a healthy limb, the plaster casts were scanned - they were made out of prosthetic gypsum. This work was performed with participation of a professional prosthetist.

The scanning data was recorded in a dedicated application for processing of point clouds, PCDMIS Reshaper, where the mirror image was also prepared. During design of the prosthesis, two other programs were used alternately, because of their complementary functions and easiness of use. CATIA v5 allowed performing the 3D modelling processes and GOM Inspect v8 was used for processing of obtained triangular mesh.

First stage of data processing was cleaning and smoothing of the obtained point clouds. Fig. 2 presents digital representation of patient's hand in the raw state and after cleaning.

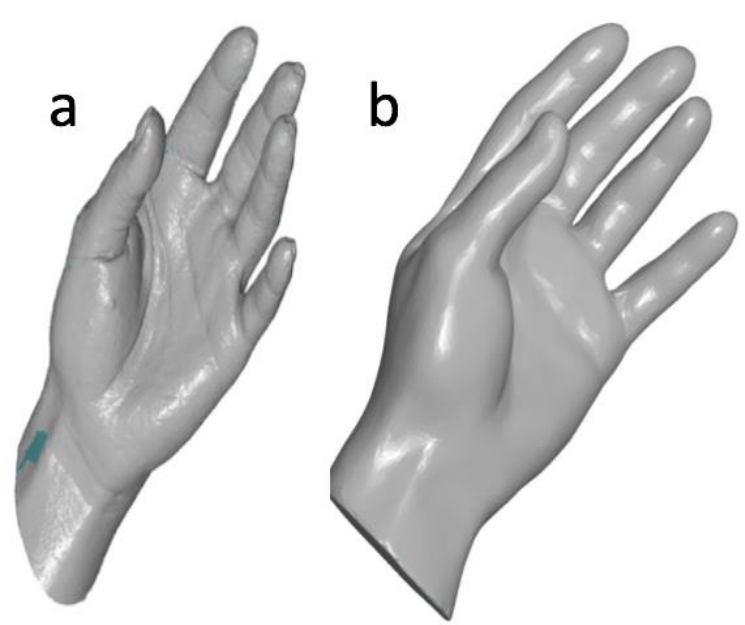

Fig. 2. Digital representation of hand before processing (a) and after processing (b) 


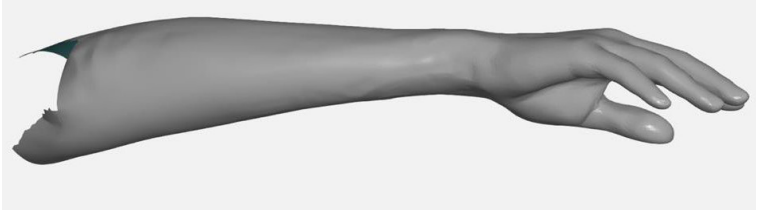

Fig. 3. Hand and forearm in a single mesh

The next step was connecting of scans of both hand and forearm in one geometry. Part of the wrist, in which the scans were connected, needed to be corrected by manual modelling. The final effect is visible in Fig. 3.

Manufacturing of the prosthesis elements was performed using a two-head FDM machine MakerBot Replicator 2X. It is a low-cost machine, which allows manufacturing out of various thermoplastic materials. An advantage of the device is its open material system - any filament of appropriate diameter (approx. 1,8mm) can be used for printing, without software or hardware limitations. Selection of this device had an impact on process of $3 \mathrm{D}$ data preparation. It would be impossible to manufacture the prosthesis in one process, as chamber of the FDM device is too small $(24,6 \mathrm{x}$ $15,2 \times 15,5 \mathrm{~cm}$ are maximal 3D print dimensions). That is why the triangle mesh was divided into 3 parts and additional holes were planned, to allow easier assembly of the final prosthesis. Division of the prosthesis is visible in Fig. 4.

For good fitting of the prosthesis socket to the patient's stump, the mesh representing the stump was subjected to an offset operation, to allow some space between the prosthesis and patient's body for ergonomic purposes (a stump can change its

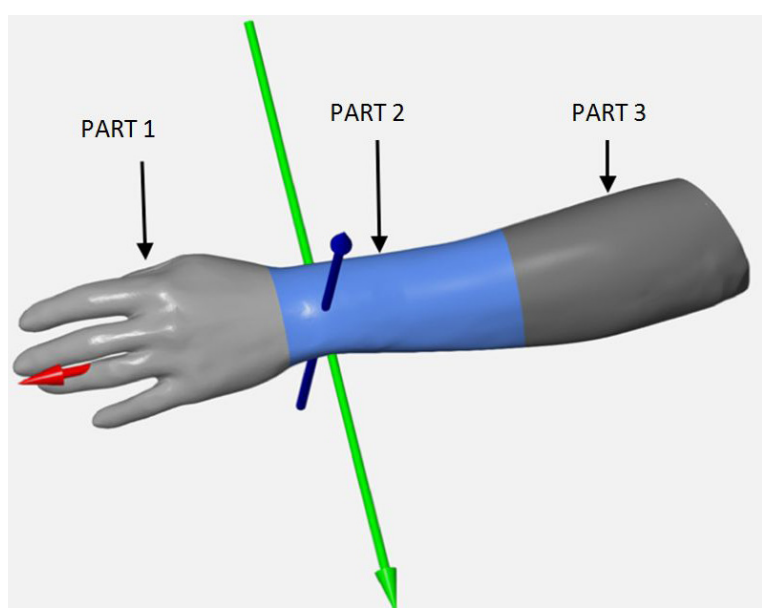

Fig. 4. Division of prosthesis into parts

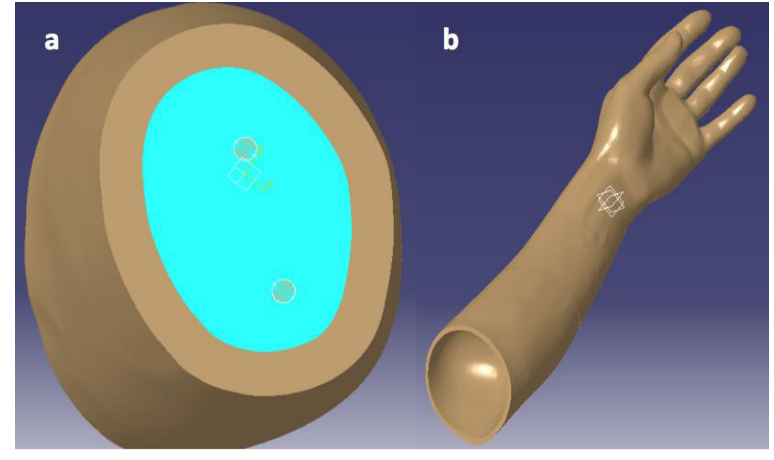

Fig. 5. Location of holes in part 3 (a), brighter color represents ABS core, darker color represents the Nin-

jaFlex shell, final 3D model of a prosthesis (b)

Table 1. Variable parameters of manufacturing for particular parts of the prosthesis.

\begin{tabular}{|c|c|c|}
\hline Part & Infill density [\%] & Raft offset [mm] \\
\hline 1 & 30 & 4 \\
\hline 2 & 28 & 4 \\
\hline 3 & 25 & 12 \\
\hline
\end{tabular}

volume, so there must be some clearance). Then, the offset scan of the stump was cut and assembled with the forearm part of the mesh. In such a way, an individualized socket was created. Fig. 5 presents part of the prosthesis with mounting holes, as well as the complete 3D model with the socket.

To make the prosthesis elastic and strong at the same time, it was decided to additionally divide it into a rigid core and an elastic shell, manufactured simultaneously in a single layer deposition process as a dual extrusion 3D print. The MakerBot Replicator 2X allows this kind of manufacturing, without any support material. For the core material, the ABS polymer was selected for its strength and rigidity. The shell was made out of filament containing thermoplastic polyurethane (TPU), sold under commercial name of NinjaFlex. The body color by name of Almond was selected to imitate natural skin. Use of the NinjaFlex also ensures good wear resistance.

On the basis of preliminary experiments, parameters of the FDM manufacturing process were selected for the dual extrusion print. No support material was planned for neither of the parts. Raft structures were planned to help sticking the parts to the table during the print. The velocities of extrusion were set as $20 \mathrm{~mm} / \mathrm{s}$ for contours and raft and $35 \mathrm{~mm} / \mathrm{s}$ for the infill. The extrusion temperature was set at $230^{\circ} \mathrm{C}$ for both materials, table temperature was $60^{\circ} \mathrm{C}$ and layer 


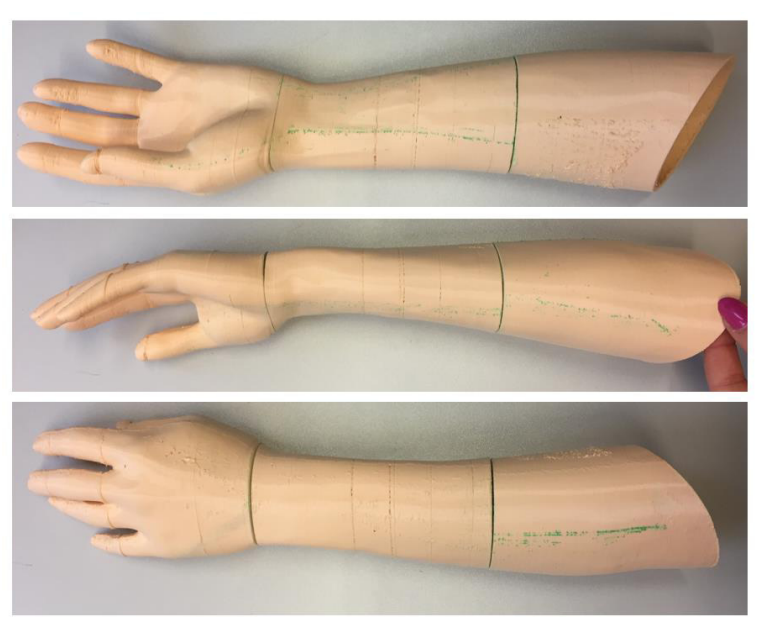

Fig. 6. Manufactured prosthesis in three views (inner forearm, side, outer forearm)

thickness was set to $0,3 \mathrm{~mm}$. The values were constant for all the parts. The parameters differing for the parts are presented in Table 1.

For the each manufactured part, post-processing was performed, using manual tools such as knife and pinch. On each part, certain deformations were detected, as well as defects in form of an excess material (both ABS and NinjaFlex), which was mostly removed by manual cutting and scraping. The assembly was performed using metal locating pins and cyanoacrylate adhesive. The assembled prosthesis is presented in Fig. 6.

\section{RESULTS AND DISCUSSION}

As a final result of the work, a functional prototype of a cosmetic prosthesis was obtained. Experimental, dual extrusion FDM process caused certain defects (visible in Fig. 7), which will be prevented in future iterations of manufacturing of this type of products by using slightly different manufacturing parameters. The authors approximated the total cost of manufacturing the prosthesis, including all the stages, according to methodology assumed in their Laboratory of Rapid Prototyping for calculation of commercial orders. The costs include:

- data gathering and processing (40 PLN/h),

- used material (NinjaFlex: 197 PLN/0,5 kg, ABS: 355 PLN/kg, plaster cast - 50 PLN total),

- time of work of 3D printer (30 PLN/h),

- time of post-processing (40 PLN/h),

- other constant costs (10 PLN).

Time of work of the reverse engineering and $3 \mathrm{D}$ printing process engineers was 27 hours in to- tal. The FDM machine was used for 40 hours. Final cost of manufacturing was approx. 2500 PLN. It is noteworthy, that the highest cost of the whole endeavor is cost of work of machine and people. Material consumption cost of 220 PLN is just a fraction of the total cost.

The making of plaster casts was difficult mostly in terms of ensuring appropriate comfort to the patient and proper mass preparation. The whole process is time-consuming and not comfortable for the patient, who needs to spend several hours at the prosthesis manufacturer's place. However, scanning of a plaster cast gives far more accurate results than scanning of a living patient.

The data preparation process in GOM Inspect v8 and CATIA v5 programs was time-consuming, because of complexity of shape of forearm and limited working area of the 3D printer. That is why appropriate division of the prosthesis and assembly features needed to be planned. Another factor, which increased data processing time, was decision of using two materials - each part of the prosthesis had to be divided into shell and core, which is not a quick thing to do if working on a triangular mesh.

The NinjaFlex material in the FDM machine was more troublesome than the ABS material. One of the persisting problems was unsticking of the semi-printed part from the modelling table. Increase of raft offset in the third part of the prosthesis helped preventing this phenomenon. Difference between the real manufacturing time and the estimated time calculated by the machine software was more than 11 hours (the real time was shorter), which may be a problem in the process planning and calculating costs. The NinjaFlex filament had certain defects and impurities - it was sticky and it did not unroll itself well from the spool. The plasticized filament put inside the heated nozzle flew through it all the time, even if it was not used (as opposed to regular materials used in the FDM process, such as ABS, PLA or HIPS). These factors caused necessity of supervising the process constantly by an operator, who had to manually unroll the filament and clean the NinjaFlex nozzle.

Post-processing of the manufactured prosthesis was problematic, as NinjaFlex is resistant to popular chemical agents (chemical smoothing was not possible using known agents, such as acetone used for chemical smoothing of ABS $3 \mathrm{D}$ prints). It is also resistant to abrasion, so no sanding or grinding could be applied with any 
good results. Thermal processing was also impossible, as models treated with a stream of hot air lose their shape. The only processing possible was manual cutting out the excess material, some of which still remained intact and visible because of green color of the used ABS.

The manufactured prototype was consulted and evaluated with participation of specialists dealing with fitting of prostheses and physiotherapy. Surface quality aside, shape of the prosthesis was evaluated very positively. An advantage of this prosthesis is that it is individual, the artificial limb is a mirror image of a real limb of the same patient, maintaining identical dimensions. Moreover, the NinjaFlex material was positively assessed in terms of imitating the human skin, regarding softness, elasticity, appropriate temperature conductivity and color. The manufactured prosthesis weighs $314 \mathrm{~g}$, which is within standard, acceptable limits of weight of this type of prosthesis, not causing discomfort and fatigue even after all-day wearing. Total manufacturing time of 5 working days (which was 3 days total, maintaining two-shift approach) is also better than time of manufacturing this type of prosthesis in a professional workshop. What's more, it was an experimental process - its repeating for another prosthesis would take considerably less time, considering higher experience of the authors.

\section{CONCLUSIONS}

The presented work allowed to state that one process of FDM can use two different materials, such as elastic NinjaFlex and rigid ABS, for manufacturing elements of cosmetic prosthesis of upper limb. Anatomically correct prototype of such a prosthesis was obtained, maintaining acceptable low mass. Softness and elasticity of skin was re-created successfully. The prosthesis was experimentally proven as strong enough to provide some support for the patient's upper body, although it is not recommended to put any heavy load on it. There were certain problems and surface errors caused by processing problems of the NinjaFlex material. However, improving of visual quality is easy, as rubber glove of body color and appropriate size can be put on the prosthesis hand, thus masking superficial defects - this method is used in professional mechanical or bionic prostheses. Still, the authors will focus their future studies on obtaining better surface quality of the extruded NinjaFlex material.
The best advantage of the applied set of methods, aside obtaining an individualized prosthesis, is low cost of manufacturing. It is a mere fraction of price, which would be paid by the patient for the traditionally made prosthesis. Presently, this method of manufacturing cosmetic limb prostheses is uncommon - use of additive manufacturing in medicine is still a new discipline, but it is growing quickly. However, it is a difficult task to perform the design process of such a product, as it requires considerable knowledge and engineering experience. The authors believe that most of the design operations for this type of product can be standardized and then automated by use of intelligent CAD models, thus greatly shortening the time of preparation of the whole product and reducing costs. This aspect will be further explored and studied by the authors in their future work.

\section{REFERENCES}

1. Biddiss E., et al. Consumer design priorities for upper limb prosthetics. Disability and Rehabilitation: Assistive Technology, 2(6), 2007, 346-357.

2. Gajdoš I., et al. Structure and tensile properties evaluation of samples produced by Fused Deposition Modeling. Open Engineering, 1(6), 2016, 86-89.

3. Górski F., et al. Application of polystyrene prototypes manufactured by FDM technology for evaporative casting method. Modern Machinery Science Journal, 2, 2017, 1729-1733.

4. Górski F., et al. Choosing optimal rapid manufacturing process for thin-walled products using expert algorithm. Journal of Industrial Engineering and Management, 3(2), 2010, 408-420.

5. Górski F., et al. Computation of mechanical properties of parts manufactured by fused deposition modeling using finite element method. Advances in Intelligent Systems and Computing, 368, 2015, 403-413.

6. Karbowski K. and Sujka W. Reverse engineering in medicine. Mechanik, 12, 2015, 111-113.

7. Kendall F., et al. Development of novel 3D-printed robotic prosthetic for transradial amputees. Prosthetics and Orthotics International, 40(3), 2016, 400-403.

8. Kłonica M. and Kuczmaszewski J. Determining the value of surface free energy on the basis of the contact angle:. Advances in Science and Technology Research Journal, 11(1), 2017, 66-74.

9. Kłonica M., et al. Polyamide 6 surface layer following ozone treatment. International Journal of Adhesion and Adhesives, 64, 2016, 179-187.

10. Pallari J., et al. Design and additive fabrication of foot and ankle-foot orthoses. Proceedings of the 
21st Annual International Solid Freeform Fabrication Symposium - An Additive Manufacturing Conference, Austin, United States 2010, 9-11.

11. Pezzin L.E., Dillingham T.R., MacKenzie E.J., Ephraim P., Rossbach P. Use and satisfaction with prosthetic limb devices and related services. Archives of Physical Medicine and Rehabilitation, 85(5), 2004, 723-729.

12. Website: Open Bionics; Ada Hand Kit; https://www. openbionics.com/shop/ada (access: 20.03.2017).

13. Website: Students develop 3-D printed exoskeletons as part of Mountaintop project; http://thebrownandwhite.com/2015/09/17/3d- printed-exoskeletons-mountaintop/ (access: 20.03.2017).

14. Website: The Agency for Health Technology Assessment and Tariff System - H72 H74 Report; http://www. aotm.gov.pl/www/wp-content/uploads/taryfikacja/2016/projekty taryf/raporty/40/WT.521.17.2016 H72_H74_raport.pdf (access: 20.03.2017).

15. Website: The limbitless arm; http://enablingthefuture.org/upper-limb-prosthetics/the-limbitlessarm/ (access: 20.03.2017).

16. Zuniga J., et al. Cyborg beast: A low-cost 3d-printed prosthetic hand for children with upper-limb differences. BMC Research Notes, 8, 2015, 10. 